\title{
Non-alcoholic fatty liver disease (NAFLD)/non-alcoholic steatohepatitis (NASH)-related liver fibrosis: mechanisms, treatment and prevention
}

\author{
Frank Tacke $^{1 \wedge}$, Ralf Weiskirchen ${ }^{2} \wedge$ \\ ${ }^{1}$ Department of Hepatology and Gastroenterology, Charité University Medicine Berlin, Berlin, Germany; ${ }^{2}$ Institute of Molecular Pathobiochemistry, \\ Experimental Gene Therapy and Clinical Chemistry (IFMPEGKC), RWTH University Hospital Aachen, Aachen, Germany \\ Contributions: (I) Conception and design: Both authors; (II) Administrative support: None; (III) Provision of study materials or patients: None; (IV) \\ Collection and assembly of data: None; (V) Data analysis and interpretation: None; (VI) Manuscript writing: Both authors; (VII) Final approval of \\ manuscript: Both authors. \\ Correspondence to: Frank Tacke, MD, PhD. Department of Hepatology and Gastroenterology, Charité University Medicine Berlin, Augustenburger \\ Platz 1, 13353 Berlin, Germany. Email: frank.tacke@charite.de.
}

\begin{abstract}
Liver fibrosis is the excessive expression and accumulation of extracellular matrix proteins in the liver. Fibrotic scarring occurs as the consequence of chronic injury and inflammation. While the successful treatment of hepatitis B and C reduced the burden of liver disease related to viral hepatitis, non-alcoholic fatty liver disease (NAFLD) or non-alcoholic steatohepatitis (NASH) are nowadays the leading causes of hepatic fibrosis worldwide. Although basic research activities have significantly advanced our understanding of the molecular disease pathogenesis, the present therapeutic options for fibrosis are still limited. In advanced disease stages, liver transplantation often remains the only curative treatment. This highlights the necessity of preventive strategies to avoid complications of fibrosis, particularly cirrhosis, portal hypertension and liver cancer. Lifestyle modifications (weight loss, exercise, healthy diet) are the basis for prevention and treatment of NAFLD-associated fibrosis. In the present review, we discuss recent advances in antifibrotic prevention and therapy. In particular, we review the current concepts for antifibrotic drug candidates in the treatment of NAFLD and NASH. While some compounds aim at reverting pathogenic liver metabolism, an alternative approach is to disconnect the injury (e.g., NAFLD) from inflammation and/or fibrosis. Investigational drugs typically target metabolic pathways, insulin resistance, hepatocyte death, inflammatory cell recruitment or activation, the gut-liver axis, matrix expression or matrix turnover. While several promising drug candidates failed in phase 2 or 3 clinical trials (including elafibranor, emricasan and selonsertib), promising results with the farnesoid $\mathrm{X}$ receptor agonist obeticholic acid, the pan-PPAR agonist lanifibranor and the chemokine receptor CCR2/CCR5 inhibitor cenicriviroc support the expectation of an effective pharmacological therapy for liver fibrosis in the near future. Tackling NAFLD-associated fibrosis from different directions by combinatorial drug treatment and effective lifestyle changes hold the greatest prospects.
\end{abstract}

Keywords: Liver fibrosis; non-alcoholic steatohepatitis (NASH); non-alcoholic fatty liver disease (NAFLD); inflammation; steatosis; clinical trials

Submitted May 29, 2020. Accepted for publication Aug 17, 2020.

doi: 10.21037/atm-20-4354

View this article at: http://dx.doi.org/10.21037/atm-20-4354

^ ORCID: Frank Tacke, 0000-0001-6206-0226; Ralf Weiskirchen, 0000-0003-3888-0931. 


\section{Introduction}

Persistent liver damage occurring in the context of metabolic (non-alcoholic fatty liver), viral hepatitis B or C, cholestatic, toxic (alcohol) or genetic (hemochromatosis, Wilson disease, etc.) diseases, usually causes a chronic inflammatory reaction. Similar to a misdirected wound healing reaction, chronic inflammation can cause scarring of the liver (1). Noteworthy, liver fibrosis is, in principle, independent of the noxious agent that causes hepatic insult, although the pattern of initial fibrosis (e.g., periportal vs. perisinusoidal) may vary. Untreated (and progressive) fibrosis can lead to cirrhosis, which is the complete architectural reconstruction with deposition of large quantities of connective tissue resulting in consecutive loss of hepatic function (2). Cirrhosis, in turn, can lead to portal hypertension, progressive liver failure and/or hepatocellular carcinoma (HCC). In recent years, it has become evident that this process is not irreversible. This offers a window of opportunity for preventive or therapeutic antifibrotic interventions.

During the past decade, the landscape of hepatology has substantially changed (3). While the effective antiviral treatment of hepatitis B and C significantly reduced the disease burden, alcohol-related liver disease remains a leading cause of liver-related mortality (4). The successful antiviral treatment against hepatitis B (viral suppression) and hepatitis C (viral eradication) provided clinical evidence that fibrosis regression can be achieved and is associated with a remarkable reduction in liver-related morbidity and mortality (5). For instance, hospital admissions related to hepatitis $\mathrm{C}$-associated liver cirrhosis have tremendously decreased, signifying the enormous clinical benefit of direct-acting antivirals (DAA) in the treatment of hepatitis C (6).

During the last years, non-alcoholic steatohepatitis (NASH) has emerged as a major etiology of liver fibrosis in Western populations, and it is expected to become the leading indication for liver transplantation and liverrelated mortality within a few years worldwide (7). Obesity, type 2 diabetes, insulin resistance, dyslipidemia, metabolic syndrome and several predisposing genetic factors (Figure 1) fuel non-alcoholic fatty liver disease (NAFLD). Alcohol-related liver disease and NAFLD are prime examples that liver fibrosis can be improved by changing life style, that means avoiding the uptake of compounds leading to the disease (i.e., alcohol) or by modifying disease drivers (overweight/obesity, insulin resistance, sedentary lifestyle). Importantly, such lifestyle-related risk factors for fatty liver disease remain relevant in patients with viral hepatitis as well, even if hepatitis $\mathrm{C}$ has been successfully cured (8).

However, alcohol-related liver disease and NAFLD are also prime examples that liver fibrosis is the hepatic manifestation of a systemic disease. In case of alcohol and NAFLD, a large number of comorbidities exist in most patients (e.g., cardiovascular diseases, diabetes, psychiatric disorders), emphasizing that prevention and treatment of liver fibrosis is only one aspect of the patient management $(9,10)$.

The substantial global burden of hepatic fibrosis to the public health systems demands the active search for effective therapies. However, despite the urgent need, approved drugs are still not available for this specific indication (11). This is majorly due to the complex molecular disturbances and the multifactorial symptoms associated with respective disease. Although lifestyle changes (particularly weight loss) are highly effective in blocking or even reversing fibrosis (12), only few patients successfully achieve a durable lifestyle change in clinical practice. Bariatric surgery is also beneficial in NASH (13), but is only available to a small fraction of morbidly obese patients. Several novel medications targeting different stages and molecular events in the disease process are currently in the pipeline, while other previously known drug candidates are used off-label or tested in clinical trials. These compounds offer new hope to patients and it is most likely that highly desirable future anti-fibrotic therapies will be established which will allow to attenuate or even reverse fibrosis and improve the quality of care in patients with liver fibrosis and cirrhosis. The histological regression of fibrosis has been accepted as a surrogate for clinically relevant endpoints in clinical trials investigating anti-NASH agents (11). This is based on the close association between fibrosis stage and "hard outcomes" including liver-related complications and mortality (14).

From a clinical perspective, it is well understood that histology is suboptimal to assess "antifibrotic" effects of an intervention (15). In cases of advanced fibrosis and cirrhosis, improving the hepatic venous pressure gradient (HVPG) might better indicate beneficial effects on portal hypertension and cirrhosis prognosis (16). Serum-based and imaging-based biomarkers may also provide information on treatment responses, but this require further prospective evaluation (17).

This review intends to summarize current concepts on strategies for the therapy and prevention of NAFLDassociated liver fibrosis. 


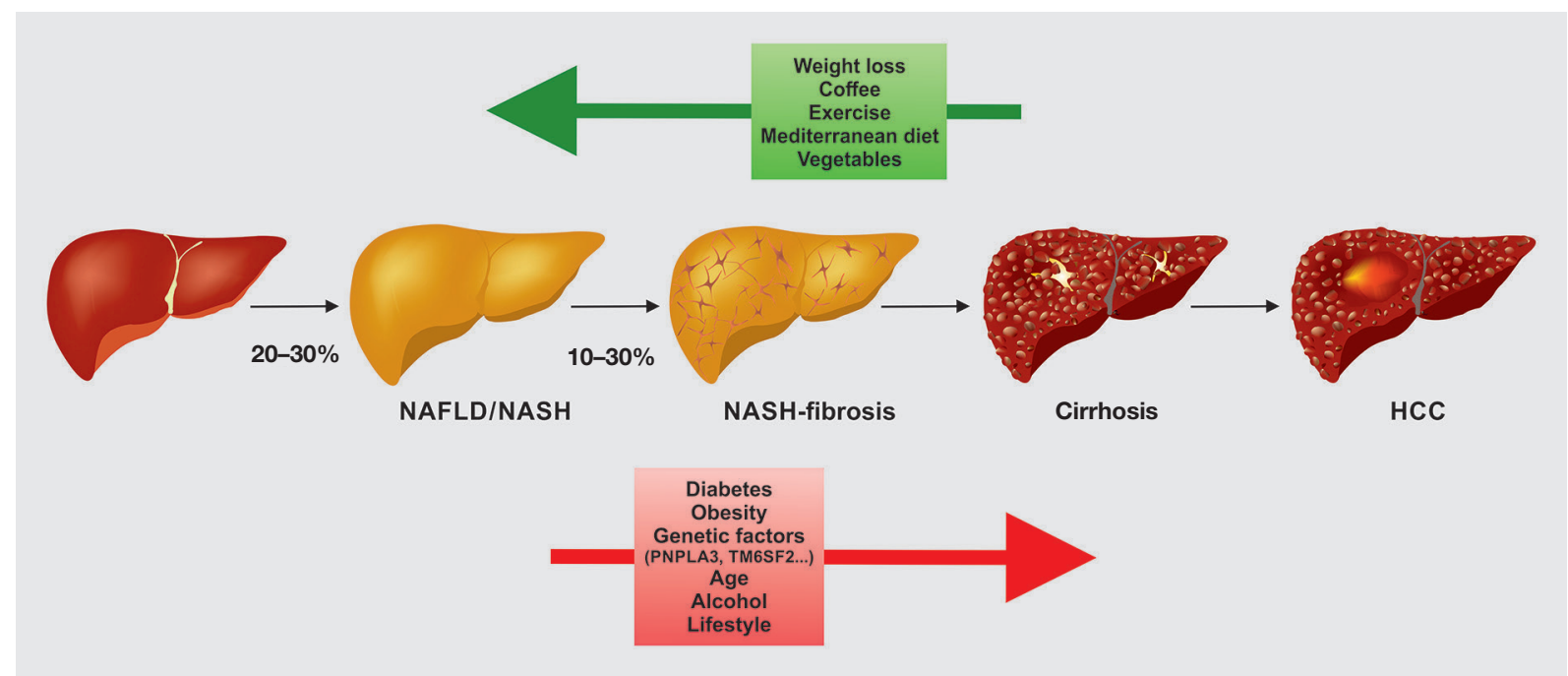

Figure 1 Clinical conditions from normal liver to NAFLD, NASH, cirrhosis, and HCC. About $25 \%$ of the general population have NAFLD, of which a substantial fraction (10-30\%) is at risk for progressing towards relevant fibrosis. Between $0.3-3 \%$ of patients with fibrosis progress to cirrhosis and/or HCC per year. The pathogenesis is influenced by many factors including genetic factors, age, diabetes, obesity, alcohol, and lifestyle. Weight loss, physical exercise, consumption of coffee, Mediterranean diet, and a vegetable-rich diet have positive effects on disease outcome and are recommended to prevent NAFLD progression. NAFLD, non-alcoholic fatty liver disease; NASH, non-alcoholic steatohepatitis; HCC, hepatocellular carcinoma.

\section{Mechanisms of liver fibrogenesis}

Fibrogenesis is initiated as a consequence of parenchymal cell damage that can be induced by different hepatotoxic agents and mechanisms. In general, an inflammatory response is initiated after tissue injury, which involves the local vascular system, the immune system, and an orchestrated local as well as systemic mobilization of soluble mediators. This response is orchestrated by nonparenchymal cells (endothelium, stellate and Kupffer cells) and resident immune cells (macrophages, dendritic cells, and mast cells) that are equipped with specialized surface receptors that recognize specific pathogen-associated molecular patterns (PAMPs) such as bacterial toxins and damage-associated molecular patterns (DAMPs). After binding, they trigger the synthesis and/or release of a large variety of different inflammatory and pro-fibrogenic mediators within the injured liver tissue. Subsequently, these soluble mediators lead to the recruitment of inflammatory immune cells into the liver tissue (18) as well as to the activation of matrix-producing cell populations driving scar tissue synthesis (1). There is an intimate crosstalk between (stressed/injured) hepatocytes, (activated) macrophages and stellate cells in the liver, which ultimately drives matrix protein deposition (19). Understanding the fibrogenic signals may offer new targets for therapeutic interventions. For instance, the recognition of liver injury by Kupffer cells, the resident phagocytes of the liver, promotes the release of chemokines including CCL2, which then promotes the recruitment of inflammatory, fibrogenic monocytes into the injured liver $(20,21)$.

The main matrix-producing cell types in liver fibrosis are hepatic stellate cells (HSCs) that transition into myofibroblasts (MFBs). Fibrogenic mediators or cell-cell contacts promote HSC activation by triggering distinct signaling cascades (22). New technologies such as singlecell RNA sequencing (scRNAseq) have helped to elucidate such activation profiles of $\mathrm{HSC} / \mathrm{MFB}$, but revealed also a striking heterogeneity among MFBs (23). For instance, HSCs partition into topographic regions, with specific differences between portal vein-associated and central vein-associated HSCs (24). Moreover, different functional aspects of HSC-e.g., chemokine/cytokine release $v s$. matrix production — can be assigned to different HSC/MFB subpopulations by scRNAseq (25). This ambiguity offers potential therapeutic options, as innovative drugs might specifically target defined myofibroblast populations (26). While targeting HSC activation and drug delivery to MFBs works already quite well in preclinical models, translation 
into human diseases has remained challenging (27).

Liver fibrogenesis is closely linked to pathological angiogenesis. Inflammatory signals, particularly from infiltrating macrophages, promote the expansion of hepatic blood vessels (28). These newly formed vessels have a different, dysfunctional, dedifferentiated phenotype compared to healthy sinusoidal endothelial cells (29). The crosstalk between pro-angiogenic endothelial cells and activated stellate cells is not only relevant during progression, but also during regression of fibrosis (30). Very likely, the fibrosis-induced angiogenesis favors the development of HCC in fibrotic livers (31). Intriguingly, changes in sinusoidal endothelial cells including loss of fenestrae ("capillarization") or the reduced ability to generate vasodilator agents occur quite early in NAFLD and can precede fibrosis, making these cells an interesting target for therapeutic interventions (32).

\section{Antifibrotic therapeutic targets}

Based on the high complexity of mediators and signaling pathways as well as the different cellular players involved in hepatic fibrogenesis, there are several new treatment options currently under evaluation. Due to the high burden of NAFLD, most antifibrotic drugs are being evaluated in patients with NASH-associated fibrosis. Liver disease in NASH is oftentimes viewed as the hepatic manifestation of the metabolic syndrome, due to the similar pathomechanisms driving obesity-associated metabolic alterations and NAFLD progression, such as oxidative stress, free fatty acids, hyperinsulinemia or systemic inflammation (33). Figure 2 provides an overview of selected therapeutic targets in NASH-related fibrosis. The different strategies can be summarized into two goals: (I) reverting pathogenic liver metabolism (in order to stop the underlying fibrosis-promoting mechanism) or (II) disconnecting NAFLD from inflammation and/or fibrosis (i.e., target "downstream" pathways so that the metabolic stress does not translate into inflammation or fibrosis).

\section{Reverting pathogenic liver metabolism}

Many metabolic risk factors contribute to the pathogenesis of NAFLD and NASH. In particular, low high-density lipoprotein (HDL), high triglycerides, hypercholesterolemia, and type 2 diabetes mellitus have been discussed as independent predictors of poor outcome in NAFLD. A recent meta-analysis of 22 population-based cohort studies suggested that type 2 diabetes and obesity as measured by body mass index (BMI) are the most important factors that are associated with more severe liver disease (35). Therefore, strategies targeting insulin resistance and diabetes are promising for ameliorating metabolic disturbances in NASH. Modern antidiabetic drugs that have a beneficial impact on weight loss may therefore also alleviate NASH. Such drug candidates are agonists of the glucagon-like peptide-1 (GLP-1), which promotes insulin secretion in a glucosedependent manner, or inhibitors of the sodium-glucose transport protein 2 (SGLT2), also called gliflozins, which inhibit glucose reabsorption in the kidney. GLP-1 receptor agonists (liraglutide, semaglutide) are currently being tested in NASH, also in non-diabetic patients. An initial pilot study demonstrated improvement of histological features of NASH by using liraglutide (36).

Besides "repurposing" anti-diabetes drugs, several strategies for targeting aspects of aberrant (liver) metabolism are in focus. Potential targets include correction of insulin resistance, interference with free fatty acid generation, triglyceride uptake, lipolysis and prevention of autophagy, endoplasmic reticulum stress, and mitochondrial functions (Figure 2, Table 1).

One key factor contributing to metabolic stress pathways are bile acid receptors such as the farnesoid $\mathrm{X}$-activated receptor (FXR) that is involved in energy expenditure and metabolism. It is involved in pathways that control lipogenesis-inducing enzymes such as the sterol regulatory element-binding transcription factor 1 (SREBP-1) and reducing endogenous bile acid production, thereby having a critical role in glucose and lipid metabolism. Drugs with agonistic FXR activity such as obeticholic acid are currently approved for the treatment of patients with primary biliary cholangitis, and was shown to be particular useful to improve insulin sensitivity in NAFLD patients suffering from type 2 diabetes $(37,38)$. These positive attributes of obeticholic acid were also confirmed in the multicenter, randomized, placebo-controlled "FXR ligand obeticholic acid in NASH treatment" (FLINT) phase 2 trial, in which NASH patients showed significant improvements in inflammation and fibrosis (39). Based on this positive finding, obeticholic acid is currently tested in a large phase 3 long-term trial in over 2,400 patients with NASH, including 2,100 patients with stage 2 or 3 liver fibrosis (40). The interim analyses from this trial are very promising, as obeticholic acid at a dose of $25 \mathrm{mg}$ daily significantly improved fibrosis and key histological components of NASH disease activity after 72 weeks of therapy (41). Based 


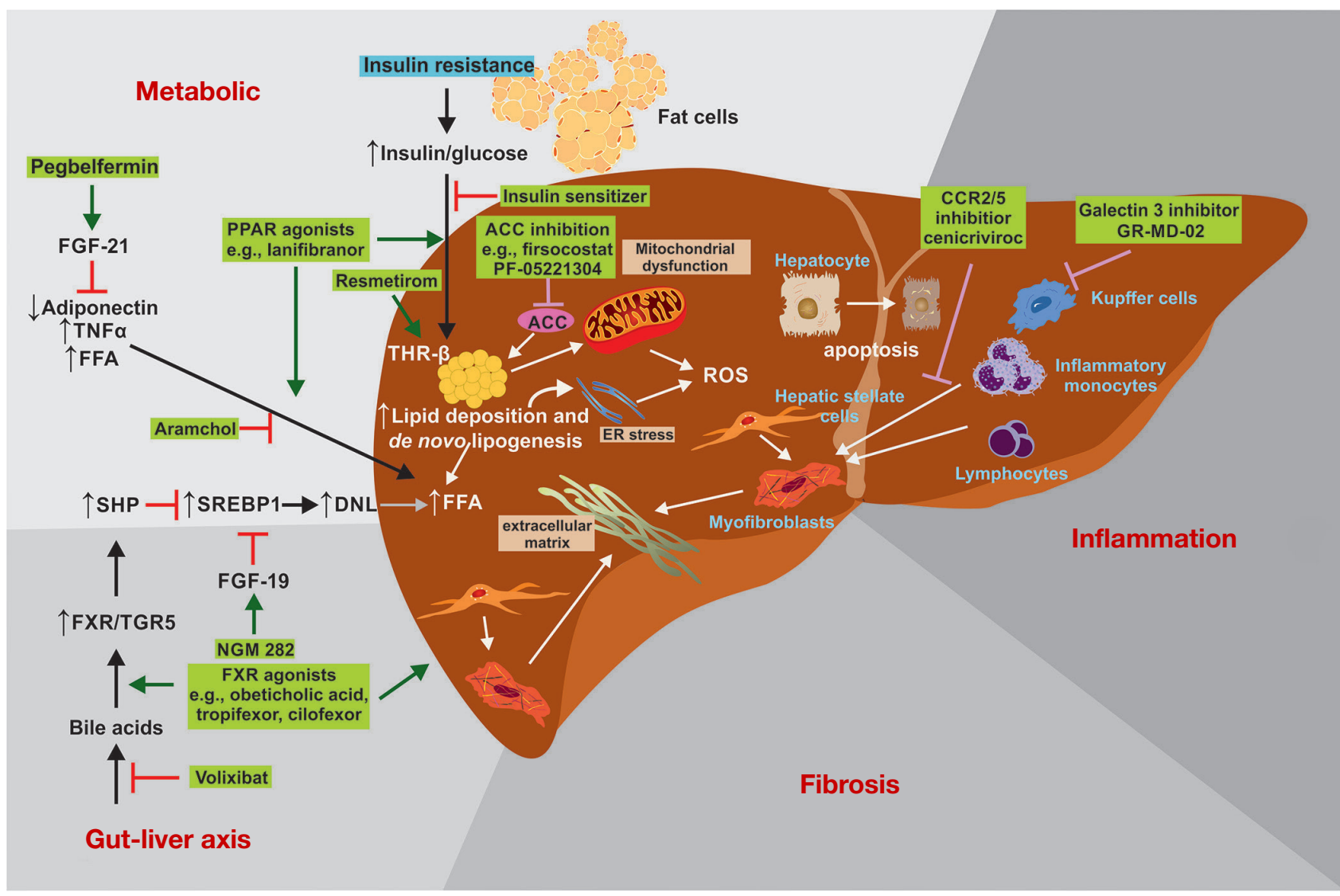

Figure 2 Therapeutic targets in liver fibrosis. The understanding of molecular processes leading to hepatic fibrosis offers emerging therapeutic targets for preventing fibrosis and enhancing therapy. Effective drugs are beneficial to prevent cell death, inflammation, and reactive oxygen species formation. Other drugs target hepatic fat metabolism, the gut-liver axis, and matrix expression or turnover. The figure is based on (34). ACC, acetyl-CoA carboxylase; DNL, de novo lipogenesis; ER, endoplasmic reticulum; FGF, fibroblast growth factor; FFA, free fatty acids; FXR, farnesoid X receptor; PPAR, peroxisome proliferator- activated receptor; ROS, reactive oxygen species; SHP, small heterodimer partner; SREBP1, Sterol regulatory element binding protein-1; TGR5, G protein-coupled bile acid receptor 1 (GPBAR1); THR $\beta$, thyroid hormone receptor- $\beta$; TNF, tumor necrosis factor.

Table 1 Selected examples for pharmacotherapies under evaluation for the treatment of NASH and/or NASH fibrosis

\begin{tabular}{|c|c|c|c|}
\hline Mechanism & Selected compounds & Effects on fibrosis & Stage of development \\
\hline ACC inhibition & Firsocostat; PF-05221304 & Possible de-activation of stellate cells & Phase 2 \\
\hline FGF19/FGF21 mimetics & $\begin{array}{l}\text { Aldafermin (FGF19); pegbelfermin } \\
\text { (FGF21) }\end{array}$ & Indirect antifibrotic effects & Phase 2 \\
\hline SCD1 inhibition & Aramchol & Indirect antifibrotic effects & Phase 2-3 \\
\hline CCR2/CCR5 inhibition & Cenicriviroc & Fibrosis regression & Phase 3 \\
\hline
\end{tabular}

$\mathrm{NASH}$, non-alcoholic steatohepatitis 
on these data, it is expected that the FDA and EMEA will conditionally approve this drug for the treatment of NASH patients with advanced fibrosis.

Many companies now developed FXR agonists without chemical similarities to bile acids. Such "non-steroidal" FXR agonist may potentially show a more favorable safety profile, as obeticholic acid has relevant side effects such as itching and elevated LDL cholesterol serum levels. Representative non-steroidal FXR agonists are tropifexor (Novartis), GS-9674 (Gilead), AKN-083 (Allergan) and LBM763 (Novartis).

Another pathway involving bile acid biology in NASH is the chemical inhibition of the apical sodium-dependent bile acid transporter (ASBT), which blocks bile acid reuptake and stimulates hepatic bile acid production. The ASBT inhibitor volixibat increased bile acid synthesis and decreased cholesterol in NASH patients, but did not show clear signals of histological benefits in a phase 2 clinical trial (42).

Other therapeutic targets are offered by the family of fibroblast growth factors (FGF) and their receptors. This growth factor family comprises 22 members that bind to five distinct receptors (FGFR1-4, FGFRL1) and signal through several downstream pathways relevant in the initiation of fibrosis (43). They have not only a substantial influence of fibrogenesis, but also impact development, regeneration, hepatocyte proliferation and recruitment of progenitor cells during acute and chronic liver injury (44). In particular, the hepatoprotective properties and beneficial effects on inflammation and fibrosis have brought FGF19 and FGF21 into the focus as novel targets for drug development. Mice lacking FGF21 showed increased hepatic fatty acid activation and $\beta$-oxidation and subcutaneous infusions of FGF2 1 reduced steatosis and peroxidative damage in a mouse NASH model (45). The PEGylated FGF21 analogue pegbelfermin (BMS-986036) demonstrated beneficial metabolic effects and reduced hepatic fat content in a 16week application in NASH patients (46). The engineered FGF19 analogue NGM282 seems to be very effective in reducing hepatic steatosis as well $(47,48)$. Its concomitant changes in circulating lipids, including increases of LDL cholesterol, can be effectively managed by coadministering rosuvastatin, as demonstrated in clinical trials (49).

An interesting group of drugs are agonists for proliferator-activated receptors (PPARs). PPARs represent a family of transcription factors that act as lipid sensors in various tissues (50). They are responsive to specific ligands, especially fatty acids, phospholipids, and prostaglandins. The family consists of three different receptors (PPAR $\alpha$,
$\mathrm{PPAR} \beta / \delta$, and $\mathrm{PPAR} \gamma$ ) that are expressed at different levels in various tissues and are targeted by different ligands. PPARs play critical roles in the regulation of liver homeostasis, fatty acid oxidation, triglyceride metabolism, and adipogenesis. They prevent fibrogenesis by keeping HSC in the quiescent phase and regulating the inflammatory response, increase insulin sensitivity and regulate triglyceride storage in adipose tissue (51). PPAR stimulation may provide therapeutic benefit against steatohepatitis and insulin resistance. Several medical compounds acting on one, two or even three PPARs already exist. Pioglitazone or rosiglitazone targeting PPAR $\gamma$ improved histological features in NAFLD (52) and may even ameliorate fibrosis (53). However, the use of these drugs in clinical routine is limited by their propensity to induce weight gain (36). In a phase 2 trial, the dual PPAR $\alpha /$ $\delta$ agonist elafibranor improved histological features and showed beneficial effects on lipid profiles, glucose, liver enzymes and inflammatory marker expression in NASH but failed to improve hepatic fibrosis (54). However, interim analyses from a phase 3 clinical trial reported that elafibranor failed to reach its primary endpoint, as elafibranor did not significantly improve the rate of patients achieving histological resolution of NASH compared to placebo.

In experimental mouse models, also the PPAR $\alpha / \gamma$ agonist saroglitazar and the triple PPAR $\alpha / \gamma / \delta$ agonist lanifibranor (IVA337) were highly effective in protecting the liver from metabolic disorders and fibrosis $(55,56)$. In mice subjected to choline-deficient high-fat diet-induced NASH, saroglitazar reduced hepatic steatosis, inflammation, ballooning and prevented fibrogenesis (55). Similar preventive and curative effects were shown for lanifibranor in preclinical mouse models of NASH (56). Most importantly, lanifibranor synergistically combined beneficial effects of agonism on PPAR $\alpha$ (on hepatocytes), PPAR $\gamma$ (on stellate cells) and PPAR $\delta$ (on macrophages) in mouse models of NASH (57). A clinical trial using lanifibranor in NASH patients is ongoing.

Improvement of NASH by increasing hepatic fat metabolism and reducing lipotoxicity was also found by usage of the liver-directed, orally active, selective thyroid hormone receptor- $\beta$ (THR $\beta$ ) agonist resmetirom (MGL3196) (58). THR $\beta$ is a nuclear hormone receptor for triiodothyronine and are associated with the control of a wide variety of effects related to development, differentiation, growth, and metabolism (59). Binding to THR $\beta$ affects metabolic processes by lowering serum lipids, cholesterol, serum triglycerides, and other metabolic 
factors. Several thyroid hormone analogues are currently under development that vary regarding selectivity (60).

Inhibition of acetyl-coenzyme carboxylase (ACC) catalyzing the rate-limiting step in de novo lipogenesis by drugs such as firsocostat (GS-0976) or PF-05221304 showed also beneficial effects on hepatic steatosis, fibrogenic marker expression and liver histology in a phase 2 randomized placebo-controlled trials of patients with NASH (61). More recent evidence suggests direct anti-fibrotic activities in addition to the beneficial metabolic effects of inhibiting ACC (62).

\section{Disconnecting NAFLD from inflammation and/or fibrosis}

It is well known that inflammation and fibrosis are key characteristics of disease progression in NAFLD. This observation fuels the concept that blocking inflammation and/or activation of MFBs or matrix deposition would provide clinically meaningful benefit to patients. Cell death and activation of inflammatory signaling pathways (in hepatocytes and non-parenchymal cells) would represent logical targets for "anti-inflammatory" interventions (63). In this direction, selonsertib, an apoptosis signal-regulating kinase 1 (ASK1) inhibitor, was proposed. In fact, selonsertib demonstrated potent antifibrotic activity in rodent models $(64,65)$. However, two large phase 3 trials in NASH patients with either advanced fibrosis or cirrhosis failed, as selonsertib did not improve liver fibrosis after 48 weeks of therapy compared to placebo (66).

One of the key findings of NASH progression towards fibrosis is the accumulation of macrophages in the liver (67). These disease-promoting fibrogenic macrophages originate from monocytes that are recruited to the liver (68) and display a unique, metabolically activated and inflammatory phenotype (69). Monocyte recruitment to NASH livers can be effectively inhibited by the chemokine receptor CCR2/CCR 5 inhibitor cenicriviroc (70). In patients with NASH and fibrosis, cenicriviroc treatment was associated with a higher rate of fibrosis improvement after 1 year of therapy (71). The antifibrotic efficacy was less clear after 2 years of cenicriviroc therapy, but this drug was exceptionally safe and well tolerated (72). A phase 3 trial evaluating cenicriviroc in NASH patients with fibrosis is currently ongoing (73).

While the clinical development of cenicriviroc in liver fibrosis is quite advanced, many other anti-inflammatory approaches have been proposed as well. For instance, blocking inflammatory macrophage activation or polarization by interfering with toll-like receptor (TLR) signaling (e.g., with the TLR4 inhibitor serelaxin) or with galectin-3 (e.g., with the inhibitor belapectin) may hold antifibrotic potential (74). Similarly, monocytes upregulate the medium chain fatty acid receptor GPR84 upon inflammatory activation. Pharmacological inhibition of GPR84 reduces myeloid cell infiltration in NASH mouse models, which subsequently ameliorates steatohepatitis and fibrosis (64). Such data underline the potential of disconnecting NAFLD from inflammation and/or fibrosis as a valid therapeutic strategy.

Targeting the activation of stellate cells directly has remained challenging. While this can be achieved in preclinical models, e.g., by innovative nanotheranostic drug delivery approaches, the translation into clinical trials is currently pending (27).

\section{The role of physical activity and lifestyle changes for treatment and prevention}

Despite the promising results from research with new pharmacological strategies, drug treatment by itself is likely not sufficient, if the unhealthy lifestyle is not corrected. Lack of physical activity is one critical factor having impact of the outcome of hepatic diseases. Undoubtedly, exercise can be very beneficial, even in advanced stages of liver fibrosis or cirrhosis (75). It has been shown that in response to exercise a large number of endogenous active mediators are produced that endorse anti-inflammatory effects (76). Therefore, it is not surprising that physical activity and aerobic exercise of moderate intensity for at least 20 to $60 \mathrm{~min}$ on at least 5 days a week in combination with resistance training performed thrice weekly has entered into the practical recommendations for the prevention and treatment of NAFLD (77).

Similarly, interventions promoting weight loss, preventing overnutrition, and reducing hepatic fat have been shown to attenuate NASH-related inflammation and fibrosis (78). Weight loss of $1 \mathrm{~kg} /$ week is recommended for overweight and obese subjects suffering from NAFLD and NASH (79). The beneficial effects of weight loss on histological features of NAFLD/NASH are well documented, and strategies to overcome barriers in adopting dietary recommendations in the management of patients have been proposed and validated (80).

Several macronutrients and micronutrients are known to either support or prevent NAFLD pathogenesis. Prototypically, fructose is a key carbohydrate that is 
associated with insulin resistance and NAFLD development, while the consumption of soluble or insoluble fibers is beneficial for the outcome of hepatic disease because they promote satiety, restrict caloric intake, slow stomach emptying, and reduce glucose and cholesterol uptake (79). Saturated fatty acids are another factor contributing to the pathogenesis of NASH/NAFLD, while the uptake of monounsaturated or polyunsaturated fatty acids has been shown to be beneficial against NAFLD (79). Among micronutrients, vitamin $\mathrm{E}$, vitamin $\mathrm{C}$, vitamin $\mathrm{D}$, and several polyphenols (e.g., resveratrol, curcumin, caffeine, quercetin) have been successfully tested in preclinical models and clinical studies $(43,79)$. They counteract intracellular reactive oxygen species formation, prevent hepatic infiltration with circulating blood cells, or target pro-inflammatory and pro-fibrotic signaling pathways and mediators that critically contribute to generation of extracellular matrix compounds (43). Nevertheless, most of these beneficial attributes of these compounds have only been shown in experimental models and the translation to humans and the initiation of human trials is perennially hampered by many factors (43).

\section{Conclusions}

Liver fibrosis, i.e., scarring of the liver as the characteristic response to chronic injury, is the main determinant of long-term outcome in patients with liver diseases. The global epidemic of obesity and the metabolic syndrome made NAFLD the leading cause of liver fibrosis to date. Lifestyle modifications, particularly weight loss, healthy diet and physical activity, are key to preventing fibrosis progression. Extensive research over the past decade has unraveled cellular and molecular mechanisms of hepatic fibrogenesis. These findings have resulted in a large number of clinical investigations on antifibrotic drugs. While several promising drug candidates failed in phase 2 or 3 clinical trials (including elafibranor and selonsertib), promising results with obeticholic acid and cenicriviroc support the expectation of an effective pharmacological therapy for liver fibrosis in the near future. To the authors' opinion, tackling NAFLD-associated fibrosis from different directions by combinatorial drug treatment and effective lifestyle changes hold the greatest prospects.

\section{Acknowledgments}

The authors thank Sabine Weiskirchen for preparing the figures and continuous support. The authors also thank the members of our labs and collaborating scientists for helpful discussions.

Funding: FT is supported by the German Research Foundation (DFG Ta434/5-1, CRC1382 Project-ID 403224013, and SFB/TRR57, P09 and Q3). RW received funding from the German Research Foundation (SFB/ TRR57, projects $\mathrm{P} 13$ and Q3) and the Interdisciplinary Centre for Clinical Research within the Faculty of Medicine at the RWTH Aachen University (IZKF Aachen, O3-1).

\section{Footnote}

Provenance and Peer Review: This article was commissioned by the Guest Editors (Ralf Weiskirchen and Wolfgang Stremmel) for the series "Unresolved Basic Issues in Hepatology" published in Annals of Translational Medicine. The article was sent for external peer review organized by the Guest Editors and the editorial office.

Conflicts of Interest: Both authors have completed the ICMJE uniform disclosure form (available at http://dx.doi. org/10.21037/atm-20-4354). The series "Unresolved Basic Issues in Hepatology" was commissioned by the editorial office without any funding or sponsorship. RW served as the unpaid Guest Editor of the series and serves as an unpaid editorial board member of Annals of Translational Medicine from Aug 2020 to Jul 2022. FT serves as an unpaid editorial board member of Annals of Translational Medicine from Aug 2020 to Jul 2022 and reports grants from Allergan, Bristol Myers Squibb, Galapagos, Gilead and Inventiva, outside the submitted work. The authors have no other conflicts of interest to declare.

Ethical Statement: The authors are accountable for all aspects of the work in ensuring that questions related to the accuracy or integrity of any part of the work are appropriately investigated and resolved.

Open Access Statement: This is an Open Access article distributed in accordance with the Creative Commons Attribution-NonCommercial-NoDerivs 4.0 International License (CC BY-NC-ND 4.0), which permits the noncommercial replication and distribution of the article with the strict proviso that no changes or edits are made and the original work is properly cited (including links to both the formal publication through the relevant DOI and the license). See: https://creativecommons.org/licenses/by-nc-nd/4.0/. 


\section{References}

1. Weiskirchen R, Weiskirchen S, Tacke F. Organ and tissue fibrosis: Molecular signals, cellular mechanisms and translational implications. Mol Aspects Med 2019;65:2-15.

2. Maurice J, Pinzani M. The stratification of cirrhosis. Hepatol Res 2020;50:535-41.

3. Karlsen TH, Tacke F. «The times they are a'changin'»Positioning the European Association for the Study of the Liver in the changing landscape of hepatology. J Hepatol 2018;68:873-5.

4. Lozano R, Naghavi M, Foreman K, et al. Global and regional mortality from 235 causes of death for 20 age groups in 1990 and 2010: a systematic analysis for the Global Burden of Disease Study 2010. Lancet 2012;380:2095-128. Erratum in: Lancet. 2013 Feb 23;381(9867):628.

5. Tacke F, Trautwein C. Mechanisms of liver fibrosis resolution. J Hepatol 2015;63:1038-9.

6. Tajes SR, Pocurull A, Castillo J, et al. Hepatitis C-related cirrhosis will be a marginal cause of hospital admissions in the near future. J Hepatol 2020;73:1360-7.

7. Estes C, Anstee QM, Arias-Loste MT, et al. Modeling NAFLD disease burden in China, France, Germany, Italy, Japan, Spain, United Kingdom, and United States for the period 2016-2030. J Hepatol 2018;69:896-904.

8. Tacke F, Boeker KHW, Klinker H, et al. Baseline risk factors determine lack of biochemical response after SVR in chronic hepatitis C patients treated with DAAs. Liver Int 2020;40:539-48.

9. Targher G, Byrne CD, Tilg H. NAFLD and increased risk of cardiovascular disease: clinical associations, pathophysiological mechanisms and pharmacological implications. Gut 2020;69:1691-705.

10. Thursz M, Kamath PS, Mathurin P, et al. Alcoholrelated liver disease: Areas of consensus, unmet needs and opportunities for further study. J Hepatol 2019;70:521-30.

11. Rinella ME, Tacke F, Sanyal AJ, et al. Report on the AASLD/EASL joint workshop on clinical trial endpoints in NAFLD. J Hepatol 2019;71:823-33.

12. Vilar-Gomez E, Martinez-Perez Y, Calzadilla-Bertot L, et al. Weight Loss Through Lifestyle Modification Significantly Reduces Features of Nonalcoholic Steatohepatitis. Gastroenterology 2015;149:367-78.e5; quiz e14-5.

13. Lassailly G, Caiazzo R, Buob D, et al. Bariatric Surgery Reduces Features of Nonalcoholic Steatohepatitis in Morbidly Obese Patients. Gastroenterology 2015;149:379-
88; quiz e15-6.

14. Taylor RS, Taylor RJ, Bayliss S, et al. Association between fibrosis stage and outcomes of patients with nonalcoholic fatty liver disease: A systematic review and meta-analysis. Gastroenterology 2020;158:1611-1625.e12.

15. Davison BA, Harrison SA, Cotter G, et al. Suboptimal reliability of liver biopsy evaluation has implications for randomized clinical trials. J Hepatol 2020;73:1322-32.

16. Garcia-Tsao G, Bosch J, Kayali Z, et al. Randomized placebo-controlled trial of emricasan for non-alcoholic steatohepatitis-related cirrhosis with severe portal hypertension. J Hepatol 2020;72:885-95.

17. Younossi ZM, Loomba R, Anstee QM, et al. Diagnostic modalities for nonalcoholic fatty liver disease, nonalcoholic steatohepatitis, and associated fibrosis. Hepatology 2018;68:349-60.

18. Heymann F, Tacke F. Immunology in the liver--from homeostasis to disease. Nat Rev Gastroenterol Hepatol 2016;13:88-110.

19. Schwabe RF, Tabas I, Pajvani UB. Mechanisms of fibrosis development in nonalcoholic steatohepatitis. Gastroenterology 2020;158:1913-28.

20. Krenkel O, Puengel T, Govaere O, et al. Therapeutic inhibition of inflammatory monocyte recruitment reduces steatohepatitis and liver fibrosis. Hepatology 2018;67:1270-83.

21. Karlmark KR, Weiskirchen R, Zimmermann HW, et al. Hepatic recruitment of the inflammatory Gr1+ monocyte subset upon liver injury promotes hepatic fibrosis. Hepatology 2009;50:261-74.

22. Tsuchida T, Friedman SL. Mechanisms of hepatic stellate cell activation. Nat Rev Gastroenterol Hepatol 2017;14:397-411.

23. Ramachandran P, Dobie R, Wilson-Kanamori JR, et al. Resolving the fibrotic niche of human liver cirrhosis at single-cell level. Nature 2019;575:512-8.

24. Dobie R, Wilson-Kanamori JR, Henderson BEP, et al. Single-cell transcriptomics uncovers zonation of function in the mesenchyme during liver fibrosis. Cell Rep 2019;29:1832-1847.e8.

25. Krenkel O, Hundertmark J, Ritz TP, et al. Single cell RNA wequencing identifies subsets of hepatic stellate cells and myofibroblasts in Liver Fibrosis. Cells 2019;8:503.

26. Yazdani S, Bansal R, Prakash J. Drug targeting to myofibroblasts: Implications for fibrosis and cancer. Adv Drug Deliv Rev 2017;121:101-16.

27. Schon HT, Bartneck M, Borkham-Kamphorst E, et al. Pharmacological intervention in hepatic stellate cell 
activation and hepatic fibrosis. Front Pharmacol 2016;7:33.

28. Ehling J, Bartneck M, Wei X, et al. CCL2-dependent infiltrating macrophages promote angiogenesis in progressive liver fibrosis. Gut 2014;63:1960-71.

29. Lefere S, Devisscher L, Geerts A. Angiogenesis in the progression of non-alcoholic fatty liver disease. Acta Gastroenterol Belg 2020;83:301-7.

30. Chen SH, Huang CL, Chiang IP, et al. Liver fibrosis regression correlates with downregulation in liver angiogenesis in chronic hepatitis $\mathrm{C}$ through viral eradication. Eur J Gastroenterol Hepatol 2020. [Epub ahead of print]. doi:10.1097/MEG.0000000000001833.

31. Bartneck M, Schrammen PL, Möckel D, et al. The CCR2+ macrophage subset promotes pathogenic angiogenesis for tumor vascularization in fibrotic livers. Cell Mol Gastroenterol Hepatol 2019;7:371-90.

32. Hammoutene A, Rautou PE. Role of liver sinusoidal endothelial cells in non-alcoholic fatty liver disease. J Hepatol 2019;70:1278-91.

33. Friedman SL, Neuschwander-Tetri BA, Rinella M, Sanyal AJ. Mechanisms of NAFLD development and therapeutic strategies. Nat Med 2018;24:908-22.

34. Konerman MA, Jones JC, Harrison SA. Pharmacotherapy for NASH: current and emerging. J Hepatol 2018;68:362-75.

35. Jarvis H, Craig D, Barker R, et al. Metabolic risk factors and incident advanced liver disease in non-alcoholic fatty liver disease (NAFLD): A systematic review and metaanalysis of population-based observational studies. PLoS Med 2020;17:e1003100.

36. Armstrong MJ, Gaunt P, Aithal GP, et al. Liraglutide safety and efficacy in patients with non-alcoholic steatohepatitis (LEAN): a multicentre, double-blind, randomised, placebo-controlled phase 2 study. Lancet 2016;387:679-90.

37. Nevens F, Andreone P, Mazzella G, et al. A placebocontrolled trial of obeticholic acid in primary biliary cholangitis. N Engl J Med 2016;375:631-43.

38. Mudaliar S, Henry RR, Sanyal AJ, et al. Efficacy and safety of the farnesoid $\mathrm{X}$ receptor agonist obeticholic acid in patients with type 2 diabetes and nonalcoholic fatty liver disease. Gastroenterology 2013;145:574-82.e1.

39. Neuschwander-Tetri BA, Loomba R, et al. Farnesoid $\mathrm{X}$ nuclear receptor ligand obeticholic acid for noncirrhotic, non-alcoholic steatohepatitis (FLINT): a multicentre, randomised, placebo- controlled trial. Lancet 2015;385:956-65.

40. Ratziu V, Sanyal AJ, Loomba R, et al. REGENERATE: Design of a pivotal, randomized, phase 3 study evaluating the safety and efficacy of obeticholic acid in patients with fibrosis due to nonalcoholic steatohepatitis. Contemp Clin Trials 2019;84:105803.

41. Younossi ZM, Ratziu V, Loomba R, et al. Obeticholic acid for the treatment of non-alcoholic steatohepatitis: interim analysis from a multicentre, randomised, placebocontrolled phase 3 trial. Lancet 2019;394:2184-96.

42. Newsome PN, Palmer M, Freilich B, et al. Volixibat in adults with non-alcoholic steatohepatitis: 24-week interim analysis from a randomized, phase II study. J Hepatol 2020;73:231-40.

43. Weiskirchen R. Hepatoprotective and anti-fibrotic agents: it's time to take the next step. Front Pharmacol 2016;6:303.

44. Ocker M. Fibroblast growth factor signaling in non-alcoholic fatty liver disease and non-alcoholic steatohepatitis: Paving the way to hepatocellular carcinoma. World J Gastroenterol 2020;26:279-90.

45. Fisher FM, Chui PC, Nasser IA, et al. Fibroblast growth factor 21 limits lipotoxicity by promoting hepatic fatty acid activation in mice on methionine and choline-deficient diets. Gastroenterology 2014;147:1073-83.e6.

46. Sanyal A, Charles ED, Neuschwander-Tetri BA, et al. Pegbelfermin (BMS-986036), a PEGylated fibroblast growth factor 21 analogue, in patients with non-alcoholic steatohepatitis: a randomised, double-blind, placebocontrolled, phase 2a trial. Lancet 2019;392:2705-17.

47. Harrison SA, Rinella ME, Abdelmalek MF, et al. NGM282 for treatment of non-alcoholic steatohepatitis: a multicentre, randomised, double-blind, placebocontrolled, phase 2 trial. Lancet 2018;391:1174-85. Erratum in: Lancet. 2018 Mar 24;391(10126):e16.

48. Harrison SA, Rossi SJ, Paredes AH, et al. NGM282 improves liver fibrosis and histology in 12 weeks in patients with nonalcoholic steatohepatitis. Hepatology 2020;71:1198-212.

49. Rinella ME, Trotter JF, Abdelmalek MF, et al. Rosuvastatin improves the FGF19 analogue NGM282-associated lipid changes in patients with non-alcoholic steatohepatitis. J Hepatol 2019;70:735-44.

50. Fougerat A, Montagner A, Loiseau N, et al. Peroxisome Proliferator-Activated Receptors and Their Novel Ligands as Candidates for the Treatment of Non-Alcoholic Fatty Liver Disease. Cells 2020;9:E1638.

51. Choudhary NS, Kumar N, Duseja A. Peroxisome Proliferator-Activated Receptors and Their Agonists in Nonalcoholic Fatty Liver Disease. J Clin Exp Hepatol 2019;9:731-9.

52. Fuchs CD, Traussnigg SA, Trauner M. Nuclear Receptor 
Modulation for the Treatment of Nonalcoholic Fatty Liver Disease. Semin Liver Dis 2016;36:69-86.

53. Musso G, Cassader M, Paschetta E, et al. Thiazolidinediones and Advanced Liver Fibrosis in Nonalcoholic Steatohepatitis: A Meta-analysis. JAMA Intern Med 2017;177:633-40. Erratum in: JAMA Intern Med. 2017 May 1;177(5):747.

54. Ratziu V, Harrison SA, Francque S, et al. Elafibranor, an Agonist of the Peroxisome Proliferator-Activated Receptor- $\alpha$ and $-\delta$, Induces Resolution of Nonalcoholic Steatohepatitis Without Fibrosis Worsening. Gastroenterology 2016;150:1147-1159.e5. Erratum in: Gastroenterology. 2017 Jun;152(8):2084.

55. Jain MR, Giri SR, Bhoi B, et al. Dual PPAR $\alpha / \gamma$ agonist saroglitazar improves liver histopathology and biochemistry in experimental NASH models. Liver Int 2018;38:1084-94.

56. Wettstein G, Luccarini JM, Poekes L, et al. The newgeneration pan- peroxisome proliferator-activated receptor agonist IVA337 protects the liver from metabolic disorders and fibrosis. Hepatol Commun 2017;1:524-37.

57. Lefere S, Puengel T, Hundertmark J, et al. Differential effects of selective- and pan-PPAR agonists on experimental steatohepatitis and hepatic macrophages. J Hepatol 2020;73:757-70.

58. Harrison SA, Bashir MR, Guy CD, et al. Resmetirom (MGL-3196) for the treatment of non-alcoholic steatohepatitis: a multicentre, randomised, double-blind, placebo-controlled, phase 2 trial. Lancet 2019;394:2012-24.

59. Gionfra F, De Vito P, Pallottini V, et al. The role of thyroid hormones in hepatocyte proliferation and liver cancer. Front Endocrinol (Lausanne) 2019;10:532.

60. Zucchi R. Thyroid Hormone Analogues: An Update. Thyroid 2020;30:1099-105.

61. Loomba R, Kayali Z, Noureddin M, et al. GS-0976 reduces hepatic steatosis and fibrosis markers in patients with nonalcoholic fatty liver disease. Gastroenterology 2018;155:1463-1473.e6.

62. Lambrecht J, Tacke F. Acetyl-CoA Carboxylase Inhibition as a Therapeutic Tool in the Battle Against NASH: Hitting More Than Just One Mechanism? Cell Mol Gastroenterol Hepatol 2020;10:859-61.

63. Schwabe RF, Luedde T. Apoptosis and necroptosis in the liver: a matter of life and death. Nat Rev Gastroenterol Hepatol 2018;15:738-52.

64. Puengel T, De Vos S, Hundertmark J, et al. The mediumchain fatty acid receptor GPR84 mediates myeloid cell infiltration promoting steatohepatitis and fibrosis. J Clin
Med 2020;9:1140.

65. Yoon YC, Fang Z, Lee JE, et al. Selonsertib Inhibits Liver Fibrosis via Downregulation of ASK1/ MAPK Pathway of Hepatic Stellate Cells. Biomol Ther (Seoul) 2020;28:527-36.

66. Harrison SA, Wong VW, Okanoue T, et al. Selonsertib for patients with bridging fibrosis or compensated cirrhosis due to NASH: Results from randomized phase III STELLAR trials. J Hepatol 2020;73:26-39.

67. Lefere S, Tacke F. Macrophages in obesity and nonalcoholic fatty liver disease: Crosstalk with metabolism. JHEP Rep 2019;1:30-43.

68. Krenkel O, Tacke F. Macrophages in nonalcoholic fatty liver disease: A role model of pathogenic immunometabolism. Semin Liver Dis 2017;37:189-97.

69. Krenkel O, Hundertmark J, Abdallah AT, et al. Myeloid cells in liver and bone marrow acquire a functionally distinct inflammatory phenotype during obesity-related steatohepatitis. Gut 2020;69:551-63.

70. Tacke F. Cenicriviroc for the treatment of non-alcoholic steatohepatitis and liver fibrosis. Expert Opin Investig Drugs 2018;27:301-11.

71. Friedman SL, Ratziu V, Harrison SA, et al. A randomized, placebo-controlled trial of cenicriviroc for treatment of nonalcoholic steatohepatitis with fibrosis. Hepatology 2018;67:1754-67.

72. Ratziu V, Sanyal A, Harrison SA, et al. Cenicriviroc Treatment for Adults With Nonalcoholic Steatohepatitis and Fibrosis: Final Analysis of the Phase 2b CENTAUR Study. Hepatology 2020;72:892-905.

73. Anstee QM, Neuschwander-Tetri BA, Wong VW, et al. Cenicriviroc for the treatment of liver fibrosis in adults with nonalcoholic steatohepatitis: AURORA Phase 3 study design. Contemp Clin Trials 2020;89:105922.

74. Chalasani N, Abdelmalek MF, Garcia-Tsao G, et al. Effects of Belapectin, an inhibitor of Galectin-3, in patients with nonalcoholic steatohepatitis with cirrhosis and portal hypertension. Gastroenterology 2020;158:1334-1345.e5.

75. Berzigotti A, Albillos A, Villanueva C, et al. Effects of an intensive lifestyle intervention program on portal hypertension in patients with cirrhosis and obesity: The SportDiet study. Hepatology 2017;65:1293-305.

76. Schon HT, Weiskirchen R. Exercise-induced release of pharmacologically active substances and their relevance for therapy of hepatic injury. Front Pharmacol 2016;7:283.

77. Oliveira CP, de Lima Sanches P, de Abreu-Silva EO, et al. Nutrition and Physical Activity in Nonalcoholic Fatty Liver Disease. J Diabetes Res 2016;2016:4597246. 
78. Moschen AR, Tilg H. Nutrition in pathophysiology and treatment of nonalcoholic fatty liver disease. Curr Opin Clin Nutr Metab Care 2008;11:620-5.

79. Ullah R, Rauf N, Nabi G, et al. Role of nutrition in the pathogenesis and prevention of non-alcoholic fatty liver disease: recent updates. Int J Biol Sci 2019;15:265-76.

80. Hallsworth K, Adams LA. Lifestyle modification

Cite this article as: Tacke F, Weiskirchen R. Non-alcoholic fatty liver disease (NAFLD)/non-alcoholic steatohepatitis (NASH)-related liver fibrosis: mechanisms, treatment and prevention. Ann Transl Med 2021;9(8):729. doi: 10.21037/atm$20-4354$ in NAFLD/NASH: Facts and figures. JHEP Rep 2019;1:468-79. 